\title{
Multidimensional approach to non-cystic fibrosis bronchiectasis: the FACED score
}

\author{
Miguel Á. Martínez-García ${ }^{1,2}$, Javier de Gracia ${ }^{2,3,4}$, Monserrat Vendrell Relat ${ }^{2,5}$, \\ Rosa-Maria Girón ${ }^{6}$, Luis Máiz Carro7, David de la Rosa Carrillo ${ }^{8}$ and \\ Casilda Olveira9
}

Affiliations: ${ }^{1}$ Pneumology Service, Hospital Universitario y Politécnico La Fe, Valencia, ${ }^{2}$ CIBER de Enfermedades Respiratorias, Banyoles, ${ }^{3}$ Pneumology Service, Hospital Vall D’Hebrón, Barcelona, ${ }^{4}$ Universidad Autónoma de Barcelona, Barcelona, ${ }^{5}$ Pneumology Service, Institut d’Investigació Biomèdica Girona, Hospital Dr Trueta, Girona, ${ }^{6}$ Pneumology Service, Hospital La Princesa, Madrid, ${ }^{7}$ Pneumology Service, Hospital Ramón y Cajal, Madrid, ${ }^{8}$ Pneumology Unit, Hospital Plató, Barcelona, and ${ }^{9}$ Pneumology Service, Hospital Universitario Regional de Málaga, Insituto de Biomedicina de Málaga, Univesidad de Málaga, Málaga, Spain.

Correspondence: M.Á. Martínez-García, Servicio de Neumología, Hospital Universitario y Politécnico La Fe, Valencia, Carrera Malilla s/n, 46006, Valencia, Spain. E-mail: mianmartinezgarcialagmail.com

ABSTRACT Bronchiectasis is a multidimensional disease and, therefore, its severity or prognosis cannot be adequately quantified by analysing one single variable. The objective of the present study was to develop a multidimensional score that classifies the severity of bronchiectasis according to its prognosis.

This is an observational multicentre study including 819 patients diagnosed with non-cystic fibrosis bronchiectasis using high-resolution computed tomography. 397 subjects were selected at random to construct the score while the remaining 422 were used for its validation. The outcome was 5 -year all-cause mortality after radiological diagnosis. A logistic regression analysis was used to select the variables included in the final score.

The final seven-point score incorporated five dichotomised variables: forced expiratory volume in $1 \mathrm{~s} \%$ predicted (F, cut-off 50\%, maximum value 2 points); age (A, cut-off 70 years, maximum value 2 points); presence of chronic colonisation by Pseudomonas aeruginosa ( $\mathrm{C}$, dichotomic, maximum value 1 point); radiological extension (E, number of lobes affected, cut-off two lobes, maximum value 1 point); and dyspnoea (D, cut-off grade II on the Medical Research Council scale, maximum value 1 point) to construct the FACED score. The validation cohort confirmed the score's validity.

We conclude that this easy-to-use multidimensional grading system proved capable of accurately classifying the severity of bronchiectasis according to its prognosis.

@ERSpublications

An easy-to-use multidimensional grading system accurately classifies bronchiectasis severity according to prognosis http://ow.ly/tW3xx

For editorial comments see page 1217.

This article has supplementary material available from www.erj.ersjournals.com

Received: Feb 112013 | Accepted after revision: Oct 272013 | First published online: Nov 142013

Support statement: This study has received a public grant from Sociedad Valenciana de Neumología (Valencian Pneumology Society) and from Praxis Pharmaceutical.

Conflict of interest: None declared.

Copyright @ERS 2014 


\section{Introduction}

Bronchiectasis is a permanent and, usually, progressive bronchial dilation resulting from the infection and chronic inflammation of the airway, leading to destruction and remodelling of the bronchial wall [1-3]. Bronchiectasis is associated with chronic and frequently purulent expectoration, multiple exacerbations and progressive, potentially disabling dyspnoea. These events gradually worsen the health-related quality of life and lung function of affected patients [4-6]. In recent years, bronchiectasis has become a major health concern for several reasons: a significant increase in the number of diagnoses $[7,8]$, the increase in the mortality rate [9] and in hospital admissions [10], the considerable health costs involved (US\$7827 per hospitalisation) [10], its negative impact on the quality of life [6] and pulmonary function of patients (annual loss of nearly $50 \mathrm{~mL}$ of forced expiratory volume in $1 \mathrm{~s}$ (FEV1)) [5] and its deleterious effects on underlying diseases [11].

Several single variables have been used to predict key outcomes of bronchiectasis, such as decline in lung function, various clinical parameters, radiological extension and the presence of chronic colonisation by Pseudomonas aeruginosa $[9,12,13]$. However, as with other airways diseases, the severity and prognosis of bronchiectasis cannot be adequately defined with one single variable. In chronic obstructive pulmonary disease (COPD), for example, some authors have noted a low correlation between symptoms and pulmonary function [14], and this has led to the construction of easy-to-use, multidimensional indices that have provided a more accurate prognosis value than the parameter normally used (FEV1 in the case of COPD) $[15,16]$. With this study, we aimed to create and validate an easy-to-use multidimensional grading system that could classify the severity of non-cystic fibrosis (CF) bronchiectasis more accurately than any single variable, according to its capacity to predict the 5-year risk of all-cause mortality.

\section{Methods}

Design

We describe an observational, multicentre study of historical cohorts involving seven Spanish centres with multidisciplinary and protocolised monographic outpatient clinics in non-CF bronchiectasis.

\section{Patients}

839 consecutive patients were included, all aged $\geqslant 18$ years and diagnosed with non-CF bronchiectasis before December 31, 2005, with a wide-ranging aetiology, radiological extension and clinical and functional impairment. Those patients whose vital state was unknown at the end of the follow-up were excluded. CF was excluded by two negative results of sweat tests conducted in those patients with bronchiectasis of unknown evident cause, or with a clinical presentation compatible with CF [17]. The study was approved by the Hospital General Universitario (Valencia, Spain) ethics and research committee (registration number 0088-89-2011).

\section{Diagnosis of bronchiectasis}

Bronchiectasis was diagnosed by high-resolution computed tomography scan of the chest in patients with a compatible clinical presentation, interpreted by specialist radiologists with extensive experience in the diagnosis of bronchiectasis. High-resolution images were obtained at full inspiration at 1-mm collimation

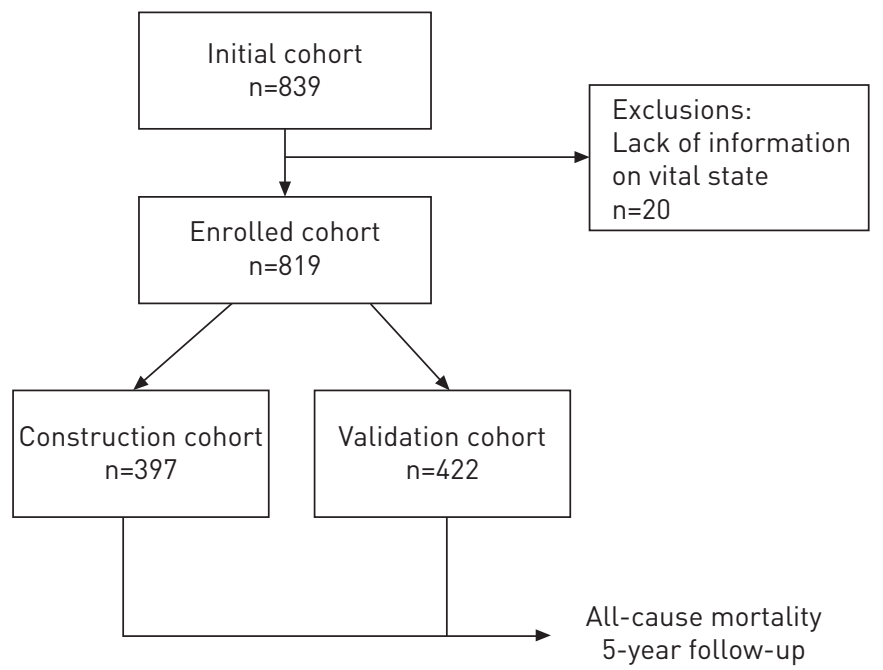

FIGURE 1 Flow chart of the study. 
TABLE 1 Comparative characteristics of the score's construction and validation cohorts

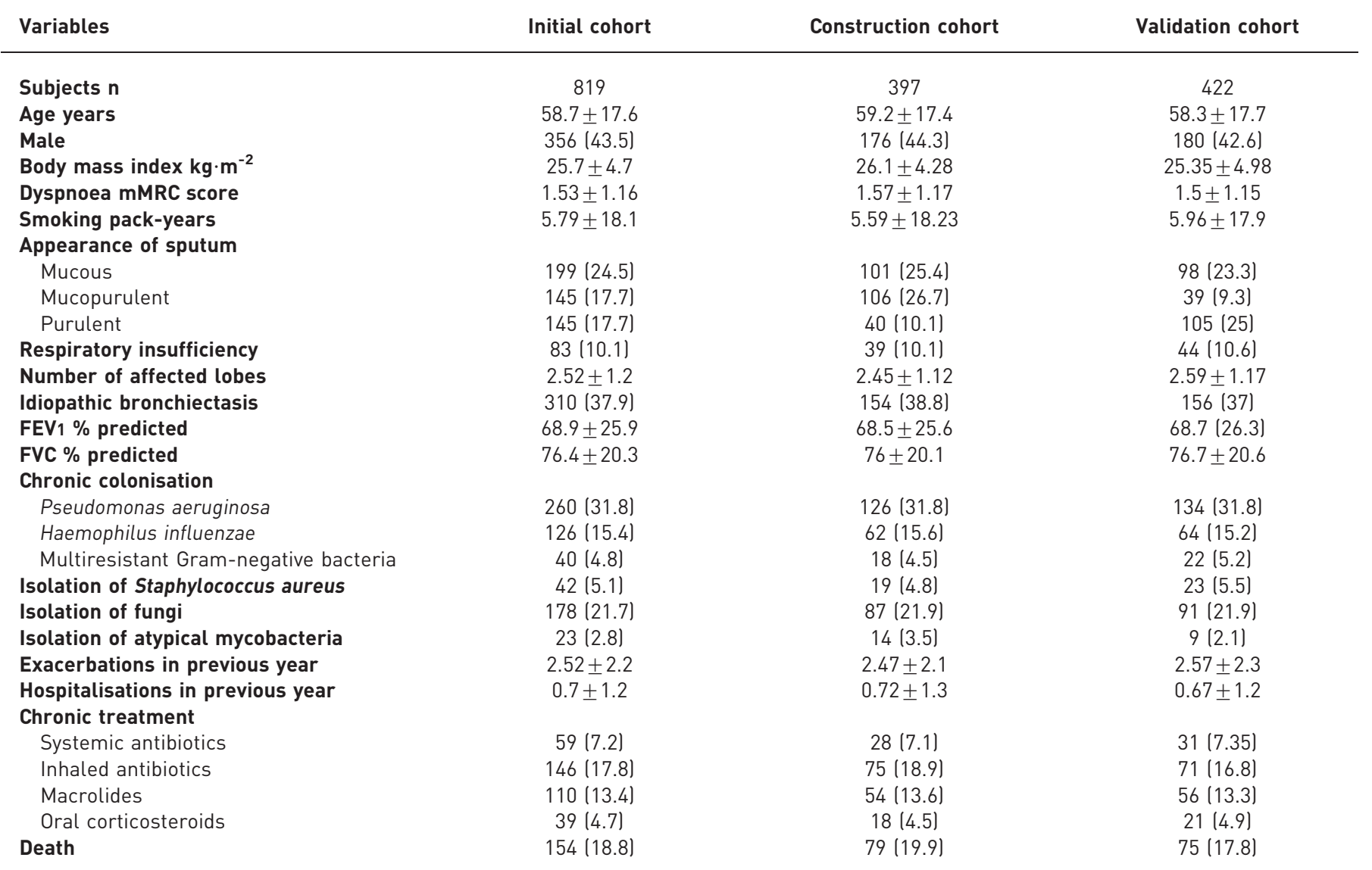

Data are presented as mean \pm SD or $n(\%)$, unless otherwise stated. mMRC: modified Medical Research Council; FEV1: forced expiratory volume in $1 \mathrm{~s} ;$ FVC: forced vital capacity.

and $10-\mathrm{mm}$ intervals from the apex to the base of the lungs. The presence of bronchiectasis was based on criteria published by NAIDICH et al. [18]. The extent of the bronchiectasis was evaluated according to the number of pulmonary lobes and segments affected, with the lingula and middle lobe considered as independent lobes. A small bronchiectasis, only visible in a single pulmonary segment, was not considered, as this can appear in a significant percentage of the healthy population, as previously reported [19].

\section{Follow-up and end-point of the study}

The vital status of all patients was determined at 5 years of follow-up, calculated from the date of radiological diagnosis of bronchiectasis. Therefore, only consecutive patients diagnosed with bronchiectasis before December 31, 2005 were considered. Death and its causes were confirmed by means of computerised hospital data or official death certificates in each centre. This follow-up period was chosen on the basis of the only long-term analysis of mortality in bronchiectasis to date, published by KeISTINEN et al. [20], who found a mortality rate of $25 \%$ after 9 years of follow-up. Accordingly, we considered that 5 years of followup from the radiological diagnosis of bronchiectasis would be sufficient to obtain a percentage of deaths with adequate statistical power for the construction and validation of the proposed score

\section{Initial selection of variables}

The same standardised protocol was used in all the centres for the diagnosis and follow-up of patients (online supplementary material). According to these criteria, 12 variables were initially eligible for the score: age, sex, body mass index, degree of dyspnoea, macroscopic appearance of sputum, number of lobes affected, chronic colonisation by $P$. aeruginosa, isolation of atypical mycobacteria, isolation of fungi, number of hospitalisations in the previous year, FEV1 \% predicted and respiratory insufficiency. 


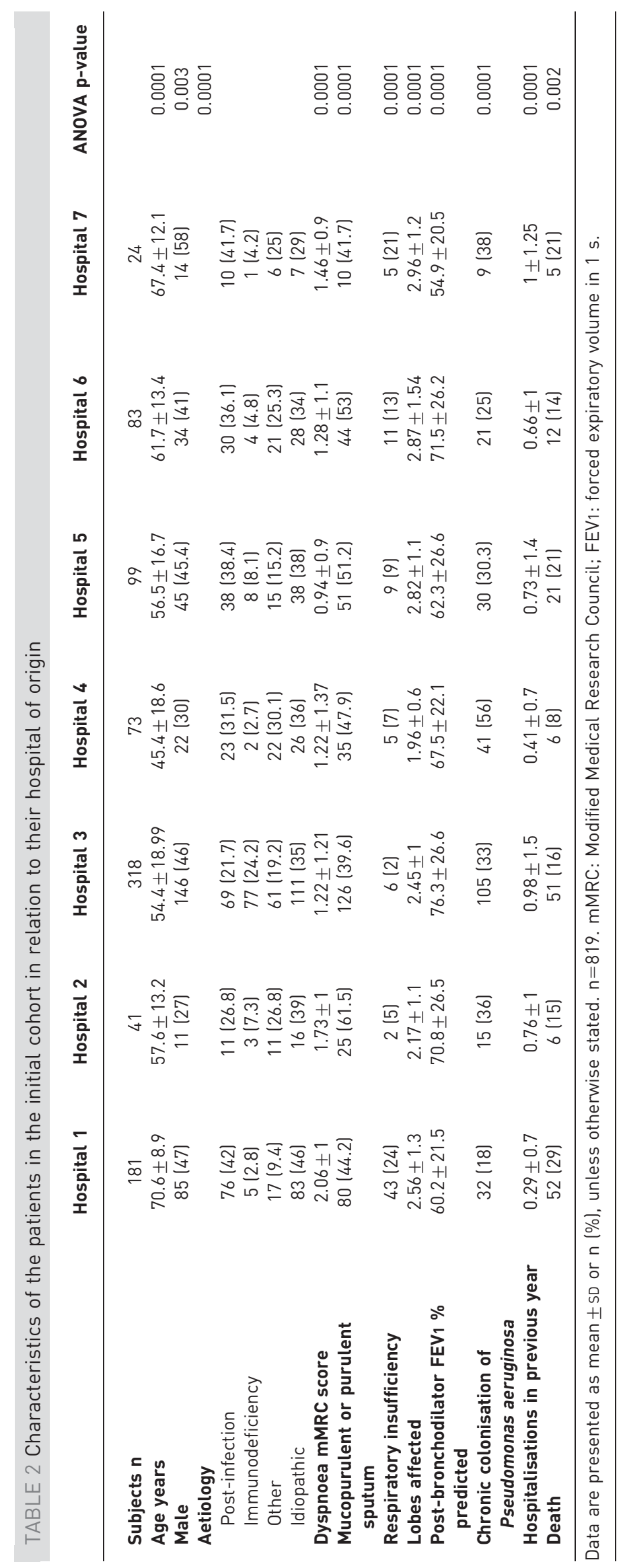


TABLE 3 Comparative characteristics of the living and dead patients after 5 years of follow-up of the construction cohort

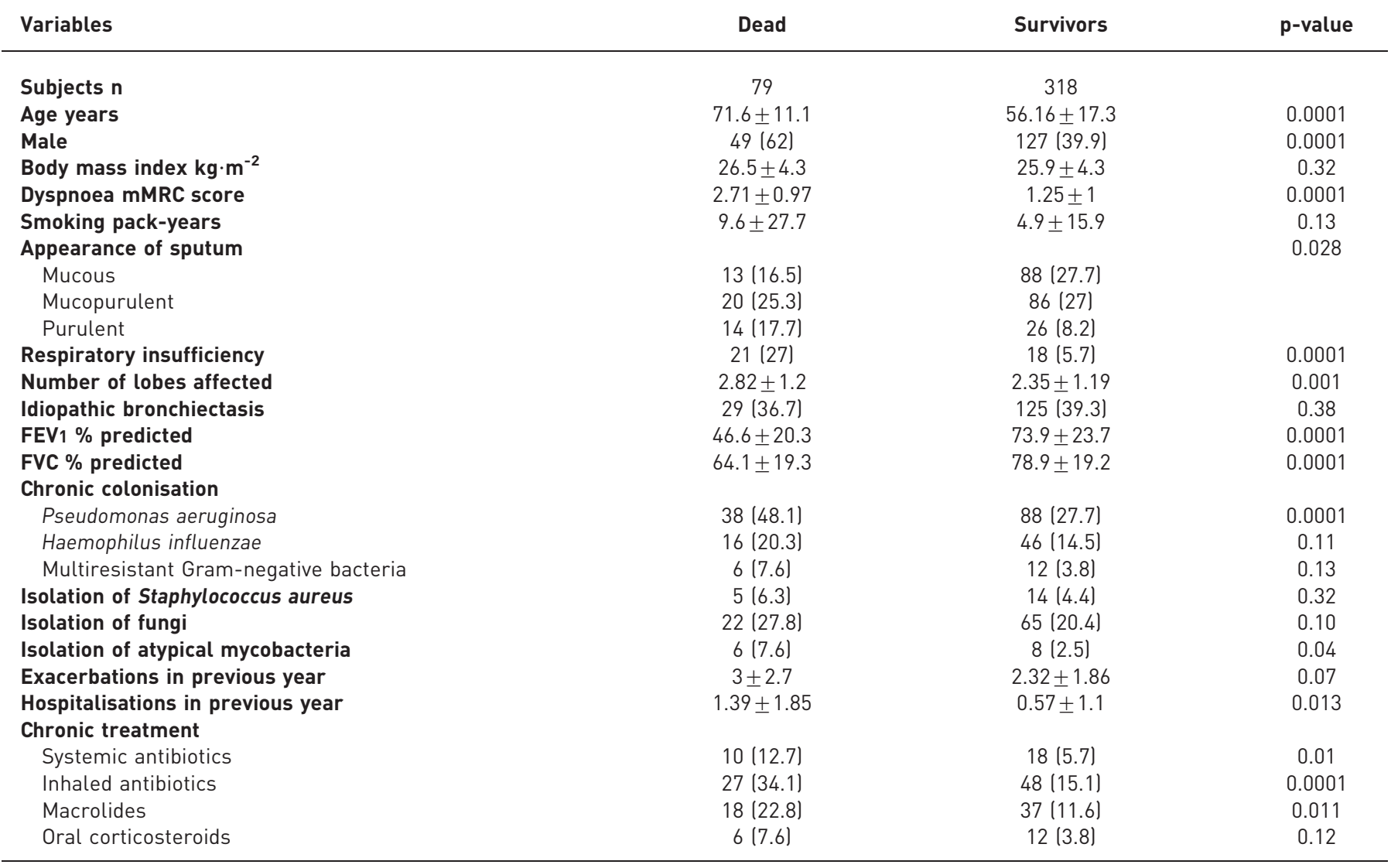

Data are presented as mean \pm SD or $\mathrm{n}(\%)$, unless otherwise stated. mMRC: modified Medical Research Council; FEV1: forced expiratory volume in $1 \mathrm{~s}$; FVC: forced vital capacity.

\section{Construction of the score}

The initial sample of 819 patients was divided randomly into two similarly sized groups by means of a computer programme (SPSS, version 17.0; IBM, Armonk, NY, USA). The first group comprised 397 patients and was used to construct the initial score (construction cohort) (fig. 1). Of the 12 variables that were initially selected, only those with a statistically significant capacity to predict the probability of death after 5 years of follow-up were chosen for the final score. Once these variables were known, they were dichotomised to facilitate the calculation of the score. We then calculated the predictive capacity of the constructed score in order to determine its validity. This would allow us to classify bronchiectasis according to its severity, on the basis of the score's capacity to predict the probability of 5-year all-cause mortality (0-2 points: mild bronchiectasis, 3-4 points: moderate bronchiectasis and 5-7 points: severe bronchiectasis).

\section{Validation of the score}

The second randomised half of the initial sample comprised 422 patients and was used to validate the constructed score (validation cohort) (fig. 1). This was evaluated by analysing whether the predictive capacity of the score using the validation cohort and its division into the three severity groups showed any significant differences when compared with the same analyses using the data from the construction cohort.

\section{Statistical analysis}

Data for the quantitative variables were tabulated as mean $\pm S D$, while the qualitative variables were tabulated as the absolute values and the percentage of the total (online supplementary material).

\section{Results}

\section{Characterisation of the sample}

20 patients were excluded due to the fact that their vital state was unknown at the end of the follow-up (fig. 1). Table 1 shows the baseline characteristics of the 819 patients finally included in the study, as well as 
TABLE 4 Predictive capacity for mortality of the 12 initial eligible variables for inclusion in the score

OR $(95 \% \mathrm{Cl})$

$1.08(1.04-1.11)$

$2.42(1.23-4.8)$

$0.96(0.94-0.97)$

$1.41(1.07-1.86)$

$1.95(1.01-3.76)$

$0.95(0.87-1.05)$

$1.64(0.82-3.25)$

$1.22(0.52-2.86)$

$1.9(0.42-8.72)$

$0.8(0.5-1.15)$

$1.18(0.95-1.45)$

$1.91(0.77-4.8)$ p-value

0.001

0.01

0.0001

0.016

0.045

0.30

0.16

0.65

0.41

0.21

0.14

0.16

Bold indicates statistical significance. mMRC: modified Medical Research Council; FEV1: forced expiratory volume in $1 \mathrm{~s}$.

the comparison between the construction $(n=397)$ and validation $(n=422)$ scores. The mean \pm SD age of the overall sample was $58.7 \pm 17.6$ years, with $32.3 \%$ aged $\geqslant=70$ years. $56 \%$ were female and $31.8 \%$ presented chronic colonisation by P. aeruginosa. There were 154 (18.8\%) deaths during follow-up, the most frequent cause being respiratory disease $(42.9 \%)$, followed by neoplasias $(9.1 \%)$ and cardiovascular disorders $(9.1 \%)$. The most frequent known aetiology of bronchiectasis was post-infectious (including posttuberculosis), in $29.3 \%$ of the cases, followed by immunodeficiency (8.3\%), COPD (3.5\%), ciliary dyskinesia (3.2\%) and systemic diseases (2.1\%). 37.9\% were of unknown aetiology. $22 \%$ of cases were cystic bronchiectasis. No statistically significant differences between the construction and validation cohorts were observed, including the aetiology of bronchiectasis (table 1).

When the patients' characteristics were compared according to their hospital of origin, significant differences were observed in relation to their general, aetiological, clinical, functional, radiological and microbiological variables (table 2). The mean \pm SD (range) follow-up was $54.7 \pm 12.9$ (1-60) months.

Univariate analysis

Table 3 shows that older age, male sex, greater degree of dyspnoea, presence of purulent sputum, respiratory insufficiency, poorer pulmonary function values, greater extension of bronchiectasis, higher number of exacerbations/hospitalisations, chronic colonisation by $P$. aeruginosa and isolation of atypical mycobacteria and fungi are related to higher mortality in the construction cohort (considering $\mathrm{p}<0.1$ as significant).

\section{Multivariate analysis: construction of the score}

Table 4 shows the results of the logistic regression analysis after including the 12 variables that were initially selected. The following showed a statistically significant association with 5-year all-cause mortality after diagnosis: age (OR 1.08, 95\% CI 1.04-1.11; p=0.001); post-bronchodilator FEV1 \% predicted (OR 0.96, 95\% CI 0.94-0.97; $\mathrm{p}=0.0001$ ); radiological extension of bronchiectasis (OR 1.41, 95\% CI: 1.07-1.86; $\mathrm{p}=0.016$ ); presence of chronic colonisation by P. aeruginosa (OR 1.95, 95\% CI 1.01-3.76; $\mathrm{p}=0.045$ ); and

TABLE 5 Predictive capacity for mortality of the different dichotomised variables included in the final score

\begin{tabular}{|c|c|c|c|c|}
\hline & OR $(95 \% \mathrm{Cl})$ & p-value & \multicolumn{2}{|c|}{$\beta$-coefficient } \\
\hline Dyspnoea mMRC score III-IV versus I-II & $2.75(1.46-5.18)$ & 0.002 & 1.01 & 1 \\
\hline $\begin{array}{l}\text { Post-bronchodilator FEV } 1<50 \% \text { versus } \geqslant 50 \% \\
\text { predicted }\end{array}$ & $5.19(2.76-9.75)$ & 0.0001 & 1.65 & 2 \\
\hline
\end{tabular}

mMRC: modified Medical Research Council; FEV1: forced expiratory volume in $1 \mathrm{~s}$. 
TABLE 6 Final score, cut-off points of the dichotomised variables and scoring of each variable

Points

$\begin{array}{lr}\text { Chronic colonisation by Pseudomonas aeruginosa } & 0 \\ \text { No } & 1 \\ \text { Yes } & \\ \text { Dyspnoea mMRC score } & 0 \\ 0-I I \\ \text { III-IV } & 1 \\ \text { FEV } 1 \% \text { predicted } & 0 \\ \geqslant 50 \% & 2 \\ <50 \% & \\ \text { Age } & 0 \\ <70 \text { years } & 2 \\ \geqslant 70 \text { years } & \\ \text { Number of lobes } & 0 \\ 1-2 & 1 \\ >2 & \end{array}$

Maximum score 7 points. mMRC: modified Medical Research Council; FEV1: forced expiratory volume in $1 \mathrm{~s}$.

dyspnoea (OR 2.42, 95\% CI 1.23-4.80; $\mathrm{p}=0.01$ ). The optimum cut-off points in the dichotomisation of the variables were as follows. Age: $>70$ years versus $\leqslant 70$ years; $\mathrm{FEV1}:>50 \%$ pred versus $\leqslant 50 \%$ pred; modified Medical Research Council (mMRC) dyspnoea score: I-II versus III-IV; radiological extension: zero to two lobes with bronchiectasis versus more than two affected lobes.

Table 5 shows the result of the logistic regression analysis, including as independent variables the five dichotomised variables that would comprise the final score called FACED. FEV1 (F, $\beta=1.65$, OR (95\% CI) 5.19 (2.76-9.75), $\mathrm{p}=0.0001)$; age (A, $\beta=1.61$, OR (95\% CI) 4.98 (2.67-9.28), $\mathrm{p}=0.0001$ ); chronic colonisation by $P$. aeruginosa (C, $\beta=0.86$, OR (95\% CI) 2.37 (1.28-4.58), $\mathrm{p}=0.006$ ); extension of bronchiectasis (E, $\beta=0.62$, OR (95\% CI) 1.87 (1.01-3.46), $\mathrm{p}=0.04)$ and dyspnoea ( $\mathrm{D}, \beta=1.01$, OR (95\% CI) $2.75(1.46-5.18), p=0.002)$. The $\beta$-coefficients were rounded in each variable to the nearest whole number to simplify the final score, as shown in table 6.

Figure 2 a shows that the area under the receiver operating characteristic curve (AUC) of the final score to predict 5-year all-cause mortality was 0.87 (the greater the AUC, the better the prognostic value of the FACED score. Any AUC $>0.8$ is considered excellent). Figure 3 a shows how the division into three scoring groups differentiated bronchiectasis into three distinct mortality groups, according to the results of the Kaplan-Meier analysis: mild (4.3\% mortality), moderate $(24.7 \%$ mortality) and severe (55.9\% mortality) bronchiectasis, given that the log-rank test marked statistically significant differences in the curves when they were compared two by two. Finally, when the AUC of the cohort of patients was calculated for each centre, it was $>0.80$ in every case apart from one centre, where it was 0.77 (range 0.77-0.92).

If we only consider the deaths from respiratory causes, the FACED score presents an AUC of 0.85 (range 0.82-0.89) and is also capable of differentiating, to a statistically significant degree, the three aforementioned groups of patients in relation to their prognosis (figure $4 \mathrm{a}$ ).

\section{Validation of the score}

Figure $2 \mathrm{~b}$ shows that the score's prognostic capacity for mortality in the validation cohort was AUC 0.83 , with no significant differences with respect to the AUC obtained with the construction cohort (C-statistics, $\mathrm{p}=0.85$ ). Similarly, no appreciable differences were observed when comparing the survival curves generated by the above mentioned division into the different scoring groups using the construction and validation cohorts (fig. 3b). Finally, using only the deaths from respiratory causes, it can be seen that the AUC is 0.86 (range 0.80-0.92), with no significant differences from the construction cohort, and that, like this cohort, the validation cohort differentiates the three prognosis groups to a statistically significant degree (fig. $4 \mathrm{~b}$ ).

\section{Discussion}

Due to the multidimensional nature of bronchiectasis, no single isolated parameter has yet proved to have sufficient power for any overall determination of its severity or prognosis. Although the measurement of quality of life could be eligible for this, its application is not generalised and the measuring instruments are not usually validated for individual use $[21,22]$. Therefore, as with other airway diseases such as COPD [14, 15] 

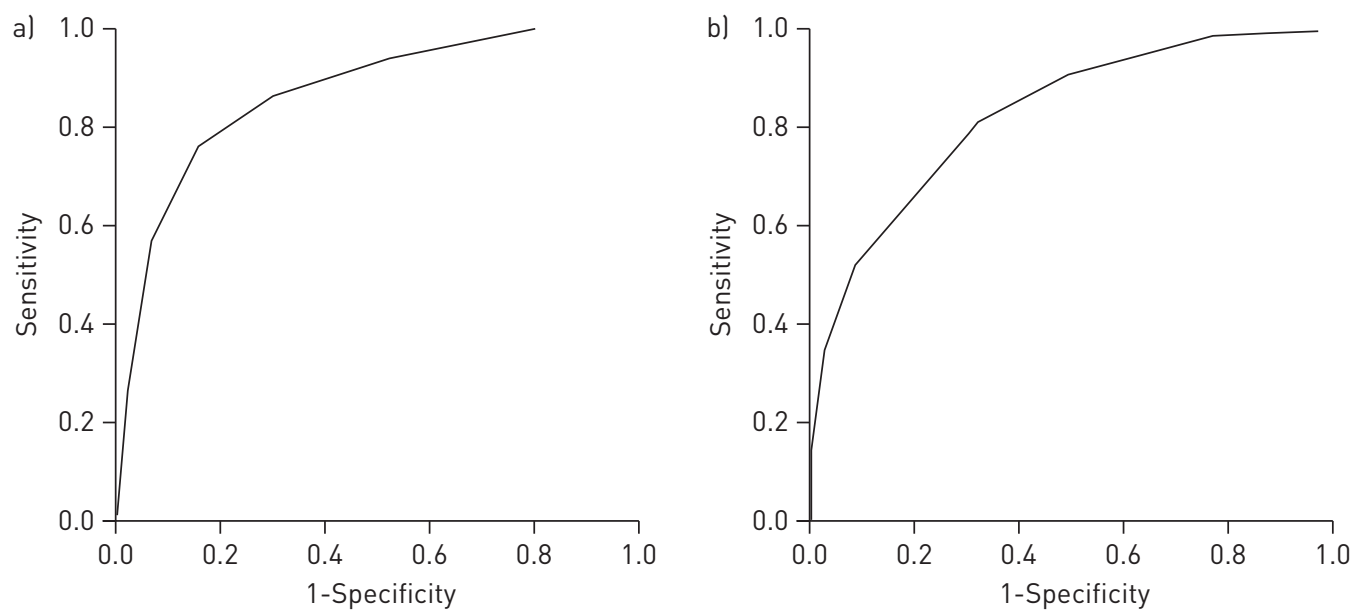

FIGURE 2 Receiver operating characteristic curves and areas under the curve (AUC) to determine the overall predictive value of mortality of the proposed score, using a) the construction cohort (AUC $0.87,95 \%$ CI $0.82-0.91$ ) and b) the validation cohort (AUC $0.83,95 \%$ CI $0.78-0.89$ ).

and infectious diseases such as pneumonia [23], it is necessary to construct and validate a score that pinpoints more effectively than any single variable the severity or prognosis of bronchiectasis by embracing the various clinical, functional, radiological and microbiological aspects characteristic of the disease. This study presents the construction and validation of a score that it is easy to calculate, obtain and interpret, while also covering all the aspects mentioned above. This score has been named FACED, an acronym for FEV1, age, chronic colonisation, extension and dyspnoea.

It should be emphasised that all the variables used for the construction and validation of the score were recorded as soon as possible after the radiological diagnosis of bronchiectasis (within a maximum of 6 months), apart from the hospitalisations, which corresponded to the year prior to a patient's admission into the study. This is crucial for the avoidance, as far as possible, of any initial interference with the various therapeutic measurements of the prognostic variables studied during the follow-up.

Of the five variables that eventually comprised the score, age and FEV1 presented the greatest predictive power of mortality. Various studies on patients with non-CF bronchiectasis have demonstrated the relationship of both these variables with an increase in mortality $[12,13]$. The high colinearity between
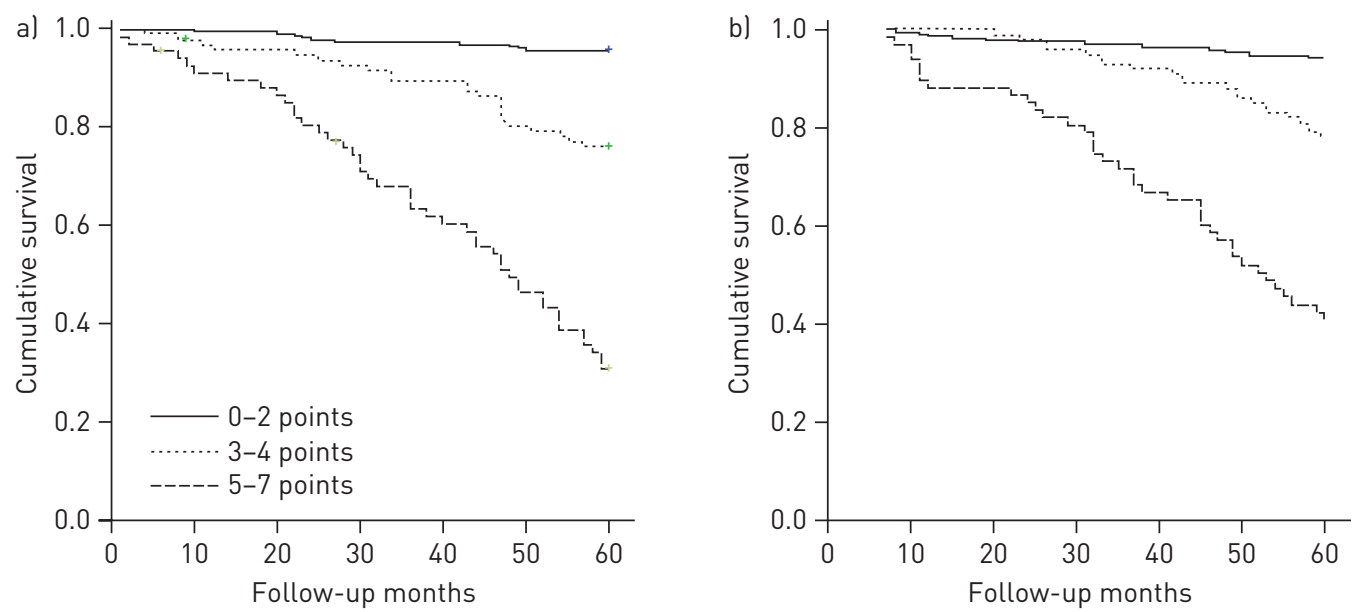

FIGURE 3 Kaplan-Meier curves for all-cause mortality corresponding to the three bronchiectasis scoring groups (mild 0-2 points, moderate 3-4 points and severe 5-7 points) in a) the construction cohort and b) the validation cohort. Logrank test a) mild bronchiectasis versus moderate bronchiectasis 29.66, $\mathrm{p}=0.0001$; mild bronchiectasis versus severe bronchiectasis 178.65, $\mathrm{p}=0.0001$; moderate bronchiectasis versus severe bronchiectasis $35.067, \mathrm{p}=0.0001$; and $\mathrm{b}$ ) mild bronchiectasis versus moderate bronchiectasis $20.02, \mathrm{p}=0.0001$; mild bronchiectasis versus severe bronchiectasis 126.81 , $\mathrm{p}=0.0001$; moderate bronchiectasis versus severe bronchiectasis $28.28, \mathrm{p}=0.0001$. a) Mild: 10 (4.3\%) out of 234 patients died; moderate: $24(24.7 \%)$ out of 98 patients died; severe: $44(68.8 \%)$ out of 64 patients died. b) Mild: 14 (5.6\%) out of 249 patients died; moderate: $22(21.6 \%)$ out of 103 patients died; severe: 38 (55.9\%) out of 68 patients died. 

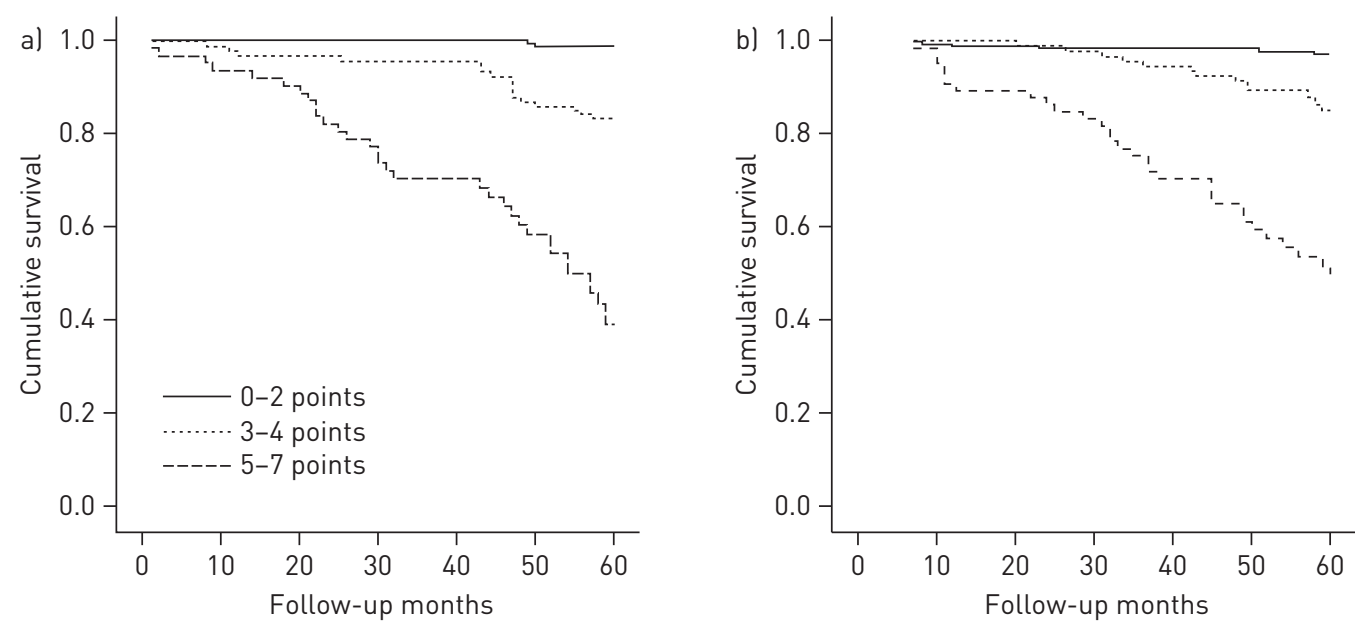

FIGURE 4 Kaplan-Meier curves for respiratory mortality corresponding to the three bronchiectasis scoring groups (mild 0-2 points, moderate 3-4 points and severe 5-7 points) in a) the construction cohort and b) the validation cohort. Logrank test a) mild bronchiectasis versus moderate bronchiectasis $32.55, \mathrm{p}=0.0001$; mild bronchiectasis versus severe bronchiectasis 178.24, $\mathrm{p}=0.0001$; moderate bronchiectasis versus severe bronchiectasis $32.84, \mathrm{p}=0.0001$; and $\mathrm{b}$ ) mild bronchiectasis versus moderate bronchiectasis $18.09, \mathrm{p}=0.0001$; mild bronchiectasis versus severe bronchiectasis 120.78 , $\mathrm{p}=0.0001$; moderate bronchiectasis versus severe bronchiectasis $26.84 ; \mathrm{p}=0.0001$. a) Mild: $2(0.9 \%)$ out of 234 patients died; moderate: 15 (15.3\%) out of 98 patients died; severe: 33 (51.6\%) out of 64 patients died. b) Mild: 6 (2.4\%) out of 249 patients died; moderate: $14(13.6 \%)$ out of 103 patients died; severe: $31(45.6 \%)$ out of 68 patients died.

FEV1 and forced vital capacity made it possible to choose FEV1 to represent the patient's functional state, as this is the variable most commonly used in the evaluation of airflow obstruction, the most common functional pattern in patients with bronchiectasis [24-26]. Although it is possible that other functional variables, such as those related to exercise tests [27], could have a greater predictive value than FEV1, they were not taken into account for the construction of this score as they are not generally applied.

The remaining variables, which represent the extension of the disease, the patient's clinical picture and microbiological aspects, also presented significant predictive power with respect to mortality, albeit to a lesser extent than age and FEV1. Dyspnoea is usually a variable with great predictive power in most studies of airway diseases, independent of lung function, and this is also the case with bronchiectasis [25], so it should not be surprising that it is one of the components of our score. It was measured using the mMRC scale [26], as this is simple and widely used. Other clinical findings were related to excess mortality in the univariate analysis (for example, the macroscopic appearance of sputum), although this was ultimately excluded from the definitive score in favour of chronic colonisation by P. aeruginosa, with which it is closely associated. Although the measurement of the quantity of sputum produced per day could be an important variable, this was rejected because it is not normally evaluated in general visits. The extension of bronchiectasis, quantified as the number of lobes affected, was incorporated into the score, even though its relative weight was lower than that of other variables. This choice is supported by authors who have found a clear dissociation between the radiological extension of bronchiectasis and its clinical and functional aspects [25], on account of the low correlation between these different variables. Other radiological variables that are more difficult to obtain and interpret, such as the characteristics of the bronchial wall, were not included, even though some studies have found them to be very important in the follow-up of these patients $[27,28]$. Lastly, one key characteristic of patients with non-CF bronchiectasis is the microbiological profile. The microbiological profiles, including fungi and atypical mycobacteria, of the patients in our study were meticulously analysed. Of all the different possible combinations, the variable that ultimately demonstrated the greatest capacity to independently predict the probability of 5-year all-cause death was the presence of chronic colonisation by $P$. aeruginosa. Neither the presence of chronic colonisation by multiresistant Gramnegative bacilli nor the isolation of Staphylococcus aureus, atypical mycobacteria and fungi, presented any significant independent predictive power for mortality. Therefore, it was finally decided to include only chronic colonisation by $P$. aeruginosa in the score, as this is the only microorganism that has been related in the current literature with an increase in mortality and a poorer functional evolution in patients with nonCF bronchiectasis [5, 29, 30].

It should be noted that the aetiology of bronchiectasis was not considered for the construction of this score, since this usually requires a series of time-consuming complementary tests that prevent the score from being calculated immediately after diagnosis, and therefore limit its application. In any case, the implementation 
of the score does not obviate the need to investigate the aetiologies of bronchiectasis, especially those that can benefit from specific treatments capable of intervening positively in its evolution [31, 32].

Finally, the predictive capacity of the FACED score was calculated when it was applied only to deaths from respiratory causes 5 years after the diagnosis of bronchiectasis, where it maintained its high predictive power (AUC $>0.80$ in both the construction and validation cohorts). We also observed a significant discriminatory capacity in the three groups of patients spanning the best and worst prognosis, again in both the construction cohort and the validation cohort.

Among the strengths of the present study, it is important to stress the very large cohort of patients included, who were well characterised as a result of their follow-up via a standardised protocol in specialised outpatient clinics by professionals with great experience in bronchiectasis. Also worthy of note are the score's multidimensional nature and the ease with which it can be obtained and interpreted, as it covers the main fields typical of patients with bronchiectasis. Lastly, an important strength of our results is their validity in all the participating centres, even though the patients from these centres presented very different characteristics and aetiologies; this indicates the robustness of the constructed score, as well as confirming its validation.

One limitation of the study is the possible influence on mortality of the various treatments administered to the patients over the 5 years of the follow-up. However, although the observational nature of our study implies the presence of limitations intrinsic to this type of methodology, no medical treatments for non-CF bronchiectasis have proved to be related to long-term mortality [31,32]. Finally, an international validation of the FACED score, including large series of patients with non-CF bronchiectasis, would be desirable.

In conclusion, our results present the construction and validation of the FACED score, which is simple to use and interpret in patients with non-CF bronchiectasis, as well as being multidimensional, on account of its assemblage of different key aspects of the disease. It shows an excellent predictive capacity for mortality and an adequate validation, independent of the varying characteristics of patients from the different participating centres, including different aetiologies, making it a robust and attractive clinical tool. New studies are required to evaluate this score's sensitivity to change by applying therapeutic variables in the follow-up of these patients.

\section{Acknowledgements}

We would like to thank A. Alvárez (Pneumology Dept, University Hospital of Vall D’Hebrón, Barcelona, Spain), G. Muñoz (Institut d'Investigació Biomèdica Girona, Hospital Dr Trueta, Girona, Spain), G. Olveira (Endocrinology and Nutrition Dept, Hospital Universitario Regional de Málaga, Instituto de Biomedicina de Málaga, Universidad de Málaga, CIBERDEM, CIBER (Instituto Carlos III: CB07/08/0019) Málaga, Spain), R. Nieto (Pneumology Dept, Ramón y Cajal Hospital, Madrid, Spain), C. Valenzuela (Pneumology Dept, La Princesa Hospital, Madrid) and P. Martínez (Pneumology Unit, Plató Hospital, Barcelona) for their participation in this study.

\section{References}

O’Donnell AE. Bronchiectasis. Chest 2008; 134: 815-823.

Barker AF. Bronchiectasis. N Engl J Med 2002; 346: 1383-1393.

Fuschillo S, De Felice A, Balzano G. Mucosal inflammation in idiopathic bronchiectasis: cellular and molecular mechanisms. Eur Respir J 2008; 31: 396-406.

4 Smith IE, Jurriaans E, Diederich S, et al. Chronic sputum production: correlations between clinical features and findings on high resolution computed tomography scanning of the chest. Thorax 1996; 51: 914-918.

5 Martínez-García MA, Soler-Cataluña JJ, Perpiñá-Tordera M, et al. Factors associated with lung function decline in adult patients with stable non-cystic fibrosis bronchiectasis. Chest 2007; 132: 1565-1572.

6 Martínez-García MA, Perpiñá-Tordera M, Román-Sánchez P, et al. Quality-of-life determinants in patients with clinically stable bronchiectasis. Chest 2005; 128: 739-745.

7 Patel IS, Vlahos I, Wilkinson TM, et al. Bronchiectasis, exacerbations indices, and inflammation in chronic obstructive pulmonary disease. Am J Respir Crit Care Med 2004; 170: 400-407.

8 Martínez-García MA, Soler-Cataluña JJ, Donat Sanz Y, et al. Factors associated with bronchiectasis in patients with COPD. Chest 2011; 140: 1130-1137.

9 García-Vidal C, Almagro P, Romaní V, et al. Pseudomonas aeruginosa in patients hospitalised for COPD exacerbation: a prospective study. Eur Respir J 2009; 34: 1072-1078.

10 Seitz AE, Olivier KN, Steiner CA, et al. Trends and burden of bronchiectasis-associated hospitalizations in the United States, 1993-2006. Chest 2010; 138: 944-949.

11 Swinson DR, Symmons D, Suresh U, et al. Decreased survival in patients with co-existent rheumatoid arthritis and bronchiectasis. Br J Reumatol 1997; 36: 689-691.

12 Onen ZP, Gulbay BE, Sen E, et al. Analysis of the factors related to mortality in patients with bronchiectasis. Respir Med 2007; 101: 1390-1397.

13 Loebinger MR, Wells AU, Hansell DM, et al. Mortality in bronchiectasis: a long-term study assessing the factors influencing survival. Eur Respir J 2009; 34: 843-849.

14 Wegner RE, Jörres RA, Kirsten DK, et al. Factor analysis of exercise capacity, dyspnoea ratings and lung function in patients with severe COPD. Eur Respir J 1994; 7: 725-729. 
15 Celli BR, Cote CG, Marín JM, et al. The body-mass index, airflow obstruction, dyspnea, and exercise capacity index in chronic obstructive pulmonary disease. N Engl J Med 2004; 350: 1005-1012.

16 Esteban C, Quintana JM, Aburto M, et al. A simple score for assessing stable chronic obstructive pulmonary disease. QJM 2006; 99: 751-759.

17 Warwick WJ, Huang NN, Waring WW, et al. Evaluation of a cystic fibrosis screening system incorporating a miniature sweat stimulator and disposable chloride sensor. Clin Chem 1986; 32: 850-853.

18 Naidich DP, McCauley DI, Khouri NF, et al. Computed tomography of bronchiectasis. J Comput Assist Tomogr 1982; 6: 437-444.

19 Lynch DA, Newell JD, Tschomper BA, et al. Uncomplicated asthma in adults: comparison of CT appearances of the lungs in asthma and healthy subjects. Radiology 1993; 188: 829-833.

20 Keistinen T, Säynäjäkangas O, Tuuponen T, et al. Bronchiectasis: an orphan disease with a poorly-understood prognosis. Eur Respir J 1997; 10: 2784-2787.

21 Murray MP, Turnbull K, MacQuarrie S, et al. Validation of the Leicester Cough Questionnaire in non-cystic fibrosis bronchiectasis. Eur Respir J 2009; 34: 125-131.

22 Wilson CB, Jones PW, O'Leary CJ, et al. Validation of the St. George's Respiratory Questionnaire in bronchiectasis. Am J Respir Crit Care Med 1997; 156: 536-541.

23 Fine MJ, Auble TE, Yealy DM, et al. A prediction rule to identify low-risk patients with community-acquired pneumonia. N Engl J Med 1997; 336: 243-250.

24 Roberts HR, Wells AU, Milne DG, et al. Airflow obstruction in bronchiectasis: correlation between computed tomography features and pulmonary function test. Thorax 2000; 55: 198-204.

25 Martínez-García MA, Perpiñá-Tordera M, Soler-Cataluña JJ, et al. Dissociation of lung function, dyspnea ratings and pulmonary extension in bronchiectasis. Respir Med 2007; 101: 2248-2253.

26 Medical Research Council Working Party. Long-term domiciliary oxygen therapy in chronic cor pulmonale complicating chronic bronchitis and emphysema. Lancet 1981; 1: 681-686.

27 Sheehan RE, Wells AU, Copley SJ, et al. A comparison of serial computed tomography and functional change in bronchiectasis. Eur Respir J 2002; 20: 581-587.

28 Lynch DA, Newell J, Hale V, et al. Correlation of CT findings with clinical evaluations in 261 patients with symptomatic bronchiectasis. AJR Am J Roentgenol 1999; 173: 53-58.

29 Evans SA, Turner SM, Bosch BJ, et al. Lung function in bronchiectasis: the influence of Pseudomonas aeruginosa. Eur Respir J 1996; 9: 1601-1604.

30 Davies G, Wells AU, Doffman S, et al. The effect of Pseudomonas aeruginosa on pulmonary function in patients with bronchiectasis. Eur Respir J 2006; 28: 974-979.

31 Vendrell M, de Gracia J, Olveira C, et al. Diagnóstico y tratamiento de las bronquiectasias. SEPAR [Diagnosis and treatment of bronchiectasis. Spanish Society of Pneumonology and Thoracic Surgery]. Arch Bronconeumol 2008; 44: 629-640.

32 Pasteur MC, Bilton D, Hill AT, et al. British Thoracic Society guideline for non-CF bronchiectasis. Thorax 2010; 65: Suppl. 1, i1-i58. 